\title{
POISSON COLOR ALGEBRAS OF ARBITRARY DEGREE
}

\author{
A.J. CALDERÓN, D.M. CHEIKH
}

\begin{abstract}
A Poisson algebra is a Lie algebra endowed with a commutative associative product in such a way that the Lie and associative products are compatible via a Leibniz rule. If we part from a Lie color algebra, instead of a Lie algebra, a graded-commutative associative product and a graded-version Leibniz rule we get a so-called Poisson color algebra (of degree zero). This concept can be extended to any degree so as to obtain the class of Poisson color algebras of arbitrary degree. This class turns out to be a wide class of algebras containing the ones of Lie color algebras (and so Lie superalgebras and Lie algebras), Poisson algebras, graded Poisson algebras, $z$-Poisson algebras, Gerstenhaber algebras and Schouten algebras among others classes of algebras. The present paper is devoted to the study of the structure of Poisson color algebras of arbitrary degree, with restrictions neither on the dimension nor the base field.
\end{abstract}

Key words: Poisson algebra, Lie color algebra, Gerstenhaber algebra, Schouten algebra, graded algebra, structure theory, simple component.

\section{INTRODUCTION}

On the one hand, we recall that Batalin-Vilkovisky (BV) formalism was introduced in physics as a way of dealing with gauge theories, being of special interest in the study of path integrals in quantum field theory. It can also be seen as a procedure for the quantization of physical systems with symmetries in the Lagrangian formalism (see [3, 13, 18]). BV formalism is just an example of application of graded Poisson algebras of integer degree. As another example, we note that it is possible to recover Hamiltonian mechanics from the coordinate space of the theory by making use of graded Poisson algebras ([20]). We can enumerate many more applications (see [2, 14, 15, 18, 23]), but we refer to [10] to a good review on this matter.

Definition 1. Let $\mathcal{P}=\bigoplus_{z \in \mathbb{Z}} \mathcal{P}_{z}$ be a $\mathbb{Z}$-graded vector space endowed with a bilinear product $\{\cdot, \cdot\}$ such that

$$
\left\{\mathcal{P}_{z}, \mathcal{P}_{z^{\prime}}\right\} \subset \mathcal{P}_{z+z^{\prime}+z_{0}}
$$

for any $z, z^{\prime} \in \mathbb{Z}$ and a fixed $z_{0} \in \mathbb{Z}$, and satisfying the identities

$$
\{x, y\}=-(-1)^{\left(|x|+z_{0}\right)\left(|y|+z_{0}\right)}\{y, x\}
$$

and

$$
\{x,\{y, z\}\}=\{\{x, y\}, z\}+(-1)^{\left(|x|+z_{0}\right)\left(|y|+z_{0}\right)}\{y,\{x, z\}\}
$$

for any homogeneous elements $x \in \mathcal{P}_{|x|}, y \in \mathcal{P}_{|y|}$ and $z \in \mathcal{P}_{|z|} . \mathcal{P}$ is called a graded Poisson algebra of degree $z_{0}$ if it is also endowed with an associative product, denoted by juxtaposition, such that

$$
\mathcal{P}_{z_{1}} \mathcal{P}_{z_{2}} \subset \mathcal{P}_{z_{1}+z_{2}}
$$

for any $z_{1}, z_{2} \in \mathbb{Z}$, and satisfies

$$
x y=(-1)^{|x||y|} y x
$$

and

$$
\{x, y z\}=\{x, y\} z+(-1)^{\left(|x|+z_{0}\right)|y|} y\{x, z\}
$$

for any $x \in \mathcal{P}_{|x|}, y \in \mathcal{P}_{|y|}$ and $z \in \mathcal{P}_{|z|}$.

In the case $z_{0}=0$ we deal with even Poisson algebras while in the case $z_{0}=1$ we are dealing with Gerstenhaber algebras.

On the other hand, we also recall that Lie color algebras were introduced in [23] as a generalization of Lie superalgebras and hence of Lie algebras. Since then, this kind of algebras has been an object of constant interest in mathematics, (see [21, 22, 32, 33, 34] for recent references), being also valuable the important role they play in theoretical physics, especially in conformal field theory and supersymmetries ([4, 16, 27, 30]). 
Definition 2. Let $\mathbb{K}$ be an arbitrary field and fix an abelian group $(G,+)$. A skew-symmetric bicharacter of $G$ is a map

satisfying

$$
\epsilon: G \times G \longrightarrow \mathbb{K} \backslash\{0\}
$$

for any $g_{1}, g_{2}, g_{3} \in G$.

$$
\begin{aligned}
\epsilon\left(g_{1}, g_{2}\right) & =\epsilon\left(g_{2}, g_{1}\right)^{-1}, \\
\epsilon\left(g_{1}, g_{2}+g_{3}\right) & =\epsilon\left(g_{1}, g_{2}\right) \epsilon\left(g_{1}, g_{3}\right),
\end{aligned}
$$

Definition 3. Let $(G,+)$ be an abelian group, $\epsilon$ a skew-symmetric bicharacter of $G$ and

$$
\mathcal{P}=\bigoplus_{g \in G} \mathcal{P}_{g}
$$

a $G$-graded $\mathbb{K}$-vector space. We shall say that $\mathcal{P}$ is a Lie color algebra if it is endowed with a bilinear product $\{\cdot, \cdot\}$ satisfying

for any $g, h \in G$, and such that

$$
\left\{\mathcal{P}_{g}, \mathcal{P}_{h}\right\} \subset \mathcal{P}_{g+h}
$$

$$
\{x, y\}=-\epsilon(|x|,|y|)\{y, x\}
$$

and

$$
\{x,\{y, z\}\}=\{\{x, y\}, z\}+\epsilon(|x|,|y|)\{y,\{x, z\}\}
$$

for any homogeneous elements $x \in \mathcal{P}_{|x|}, y \in \mathcal{P}_{|y|}$ and $z \in \mathcal{P}_{|z|}$.

Lie superalgebras (and so Lie algebras) are examples of Lie color algebras by considering $G=\mathbb{Z}_{2}$ and $\epsilon(i, j)=(-1)^{i j}$, for any $i, j \in \mathbb{Z}_{2}$.

Now we have to note that another class of Poisson-type algebras similar to the one of graded Poisson algebras of degree $z_{0}$ in Definition 1 but replacing the group $\mathbb{Z}$ by $\mathbb{Z}_{2}$ has been considered in the literature. This kind of algebras are known as even and odd Poisson superalgebras, depending on taking degree $\overline{0}$ or degree $\overline{1}$, being of interest in studying, for instance, two-dimensional supergravity and three-dimensional systems ([2, 11, 14, 23]). However, as we know, there is not a category in the literature which allows us to combine a graded bracket of degree $g_{0} \in G$ and a graded commutative associative product via a graded Leibniz identity when the group $G$ is an arbitrary abelian group. In the present paper we will introduce such a notion by starting from a degree $g_{0}$ generalization of a Lie color algebra.

Definition 4. Let $(G,+)$ be an abelian group, $\epsilon$ a skew-symmetric bicharacter of $G$ and

$$
\mathcal{P}=\bigoplus_{g \in G} \mathcal{P}_{g}
$$

a $G$-graded $\mathbb{K}$-vector space endowed with a bilinear product $\{\cdot, \cdot\}$ satisfying

$$
\left\{\mathcal{P}_{g}, \mathcal{P}_{h}\right\} \subset \mathcal{P}_{g+h+g_{0}}
$$

for any $g, h \in G$ and a fixed $g_{0} \in G$, and such that

$$
\{x, y\}=-\epsilon\left(|x|+g_{0},|y|+g_{0}\right)\{y, x\} \quad \text { (Anticonmutativity) }
$$

and

$$
\{x,\{y, z\}\}=\{\{x, y\}, z\}+\epsilon\left(|x|+g_{0},|y|+g_{0}\right)\{y,\{x, z\}\} \quad \text { (Jacobi Identity) }
$$

for any homogeneous elements $x \in \mathcal{P}_{|x|}, y \in \mathcal{P}_{|y|}$ and $z \in \mathcal{P}_{|z|}$. It is said that $\mathcal{P}$ is a Poisson color algebra of degree $g_{0}$, if it is also endowed with an associative product, denoted by yuxtaposition, such that

$$
\mathcal{P}_{g} \mathcal{P}_{h} \subset \mathcal{P}_{g+h}
$$

for any $g, h \in G$, and satisfies

$$
x y=\epsilon(|x|,|y|) y x \quad \text { (Conmutativity) }
$$

and

$$
\{x, y z\}=\{x, y\} z+\epsilon\left(|x|+g_{0},|y|\right) y\{x, z\} \quad \text { (Leibniz Identity) }
$$

for any $x \in \mathcal{P}_{|x|}, y \in \mathcal{P}_{|y|}$ and $z \in \mathcal{P}_{|z|}$. 
This class of algebras turns out to be a wide one containing those of Lie color algebras (and so Lie superalgebras and Lie algebras), Poisson algebras, graded Poisson algebras, $z$-Poisson algebras, Gerstenhaber algebras ([1, 29, 31]), and Schouten algebras ([5, 12, 19]), among other classes of algebras, being these classes of algebras of increasing interest in mathematical physics, especially in Hamiltonian and Lagrangian dynamics and mechanics. Hence Poisson color algebras of degree $g_{0}$ allow us to treat all of these classes of algebras from a common view point and extend their formalisms to non-necessarily $\mathbb{Z}$-graded or $\mathbb{Z}_{2}$-graded contexts. We also note that the case of degree 0 has been previously considered in [28] for the case of Banach algebras, in the study of a color extension of Hamiltonian formalism. Also a geometric approach to the ideas of [28] can be found in [19], where it is presented a Poisson geometry in this context.

The usual regularity concepts will be understood in the graded sense. That is, a subalgebra of a Poisson color algebra $\mathcal{P}$ of arbitrary degree is a graded linear subspace $\mathcal{Q}$ satisfying $\{\mathcal{Q}, \mathcal{Q}\}+\mathcal{Q} \mathcal{Q} \subset \mathcal{Q}$. An ideal $\mathcal{I}$ of $\mathcal{P}$ is a subalgebra satisfying $\{\mathcal{I}, \mathcal{P}\}+\{\mathcal{P}, \mathcal{I}\}+\mathcal{I} \mathcal{P}+\mathcal{P} \mathcal{I} \subset \mathcal{I}$. Finally, $\mathcal{P}$ is called simple if $\{\mathcal{P}, \mathcal{P}\} \neq 0, \mathcal{P} \mathcal{P} \neq 0$ and its only ideals are $\{0\}$ and $\mathcal{P}$.

We are interested in the present paper in studying the structure of Poisson color algebras $\mathcal{P}$ of arbitrary degree. The paper is organized as follows. In $\S 2$ we develop techniques of connections in the restricted support of $\mathcal{P}$ so as to show that $\mathcal{P}$ is of the form $\mathcal{P}=U+\sum_{j} \mathcal{I}_{j}$ with $U$ a linear subspace of $\mathcal{P}_{0}+\mathcal{P}_{g_{0}}+\mathcal{P}_{-g_{0}}$ and any $\mathcal{I}_{j}$ a well described (graded) ideal of $\mathcal{P}$, satisfying $\left\{\mathcal{I}_{j}, \mathcal{I}_{k}\right\}+\mathcal{I}_{j} \mathcal{I}_{k}=0$ if $j \neq k$. In $\S 3$, and under mild conditions, the simplicity of $\mathcal{P}$ is characterized and it is shown that any Poisson color algebra $\mathcal{P}$ of arbitrary degree is the direct sum of the family of its minimal (graded) ideals, each one being a simple Poisson color algebra of the same degree.

Finally we note that, throughout this paper, Poisson color algebras of degree $g_{0} \in G$ are considered of arbitrary dimension and over an arbitrary base field $\mathbb{K}$.

\section{CONNECTIONS AND GRADINGS}

In the following,

$$
\mathcal{P}=\bigoplus_{g \in G} \mathcal{P}_{g}
$$

denotes a Poisson color algebra of degree $g_{0}$. We will write by

$$
\Sigma=\left\{g \in G: \mathcal{P}_{g} \neq 0\right\} \backslash\left\{0, \pm g_{0}\right\}
$$

the restricted support of $\mathcal{P}$ and by

$$
-\Sigma=\{-g: g \in \Sigma\} \subset G \backslash\left\{0, \pm g_{0}\right\}
$$

Definition 5. Let $g$ and $h$ be two elements in $\Sigma$. We shall say that $g$ is connected to $h$ if there exist

$$
g_{1}, g_{2}, \ldots, g_{n} \in \pm \Sigma \cup\left\{0, \pm g_{0}\right\} \text { and } k_{2}, k_{3}, \ldots, k_{n} \in\left\{0, \pm g_{0}\right\}
$$

such that:

1. $g_{1}=g$,

2. $g_{1}+g_{2}+k_{2} \in \pm \Sigma$,

$g_{1}+g_{2}+k_{2}+g_{3}+k_{3} \in \pm \Sigma$,

$g_{1}+g_{2}+k_{2}+g_{3}+k_{3}+g_{4}+k_{4} \in \pm \Sigma$,

.........

$g_{1}+g_{2}+k_{2}+g_{3}+k_{3}+\cdots+g_{n-1}+k_{n-1} \in \pm \Sigma$,

4. $g_{1}+g_{2}+k_{2}+g_{3}+k_{3}+\cdots+g_{n}+k_{n}=\epsilon h$ for some $\epsilon \in \pm 1$.

We shall also say that

$$
\left\{g_{1} \otimes 0, g_{2} \otimes k_{2}, g_{3} \otimes k_{3}, \ldots, g_{n} \otimes k_{n}\right\}
$$

is a connection from $g$ to $h$.

Proposition 1. The relation $\sim$ in $\Sigma$, defined by $g \sim h$ if and only if $g$ is connected to $h$ is an equivalence relation. 
Proof. The set $\{g \otimes 0\}$ is a connection from $g$ to itself and therefore $g \sim g$.

If $g \sim h$ and $\left\{g_{1} \otimes 0, g_{2} \otimes k_{2}, g_{3} \otimes k_{3}, \ldots, g_{n} \otimes k_{n}\right\}$ is a connection from $g$ to $h$, then it is straightforward to verify that

$$
\left\{h \otimes 0,-\epsilon g_{n} \otimes-\epsilon k_{n},-\epsilon g_{n-1} \otimes-\epsilon k_{n-1}, \ldots,-\epsilon g_{3} \otimes-\epsilon k_{3},-\epsilon g_{2} \otimes-\epsilon k_{2}\right\}
$$

is a connection from $h$ to $g$ in case

$$
g_{1}+g_{2}+k_{2}+g_{3}+k_{3}+\cdots+g_{n-1}+k_{n-1}+g_{n}+k_{n}=\epsilon h .
$$

Therefore $h \sim g$.

Finally, suppose $g \sim h$ and $h \sim l$, and write

$$
\left\{g_{1} \otimes 0, g_{2} \otimes k_{2}, \ldots, g_{n} \otimes k_{n}\right\}
$$

for a connection from $g$ to $h$ and $\left\{h_{1} \otimes 0, h_{2} \otimes k_{2}^{\prime}, \ldots, h_{m} \otimes k_{m}^{\prime}\right\}$ for a connection from $h$ to $l$.

If $m=1$, then $l \in\{ \pm h\}$ and so the own connection (1) gives us $g \sim l$.

If $m>1$, then it is easy to check that

$$
\left\{g_{1} \otimes 0, g_{2} \otimes k_{2}, \ldots, g_{n} \otimes k_{n}, \epsilon h_{2} \otimes \epsilon k_{2}^{\prime}, \epsilon h_{3} \otimes \epsilon k_{3}^{\prime}, \ldots, \epsilon h_{m} \otimes \epsilon k_{m}^{\prime}\right\}
$$

is a connection from $g$ to $l$ in case $g_{1}+g_{2}+k_{2}+g_{3}+k_{3}+\cdots+g_{n}+k_{n}=\epsilon h$. Therefore $g$ is connected to $l$ and $\sim$ is an equivalence relation.

By Proposition 1 the connection relation is an equivalence relation in $\Sigma$ and so we can consider the quotient set

$$
\Sigma / \sim=\{[g]: g \in \Sigma\},
$$

becoming $[g]$ the set of elements in the restricted support of the grading which are connected to $g$.

Remark 1. Observe that for any $g \in \Sigma$, if $\epsilon g+\mu g_{0} \in \Sigma$ for some $\epsilon \in \pm 1$ and some $\mu \in\{0\} \cup\{ \pm 1\} \cup\{ \pm 2\}$ then

$$
\epsilon g+\mu g_{0} \in[g] .
$$

Indeed, we just have to consider either the connection $\left\{g \otimes 0,0 \otimes \epsilon \mu g_{0}\right\}$ when $\mu \in\{0\} \cup\{ \pm 1\}$, or $\left\{g \otimes 0, g_{0} \otimes g_{0}\right\}$ when $\mu=2 \epsilon$, or $\left\{g \otimes 0,-g_{0} \otimes-g_{0}\right\}$ when $\mu=-2 \epsilon$.

Our final goal in this section is to associate an adequate subalgebra $\mathcal{I}_{[g]}$ to any $[g] \in \Sigma / \sim$.

Fix $g \in \Sigma$, we start by defining the following linear subspaces. For any

$$
\alpha \in\left\{0, g_{0},-g_{0}\right\}
$$

let us write

$$
\sum_{\left\{h \in[g], p \in \Sigma: p=-h-g_{0}+\alpha\right\}}\left\{\mathcal{P}_{\alpha,[g]}:=\right.
$$

Observe that whence $h \in[g]$ and $-h-g_{0}+\alpha \in \Sigma$, (resp. $k \in[g]$ and $-k+\alpha \in \Sigma$ ), then the connection $\left\{h \otimes 0, g_{0} \otimes-\alpha\right\}$, (resp, $\{k \otimes 0,-\alpha \otimes 0\}$ ), together with the transitivity of the connection relation, give us $-h-g_{0}+\alpha \in[g]$, (resp. $-k+\alpha \in[g]$ ). Also observe that the possibility $q=-g_{0}$ just holds when $\alpha=g_{0}$ and $k=2 g_{0} \in \Sigma$. Next we define

$$
\mathcal{V}_{[g]}:=\bigoplus_{h \in[g]} \mathcal{P}_{h}
$$

Finally, we denote by $\mathcal{I}_{[g]}$ the direct sum

$$
\mathcal{I}_{[g]}:=\left(\sum_{\alpha \in\left\{0, g_{0},-g_{0}\right\}} \mathcal{P}_{\alpha,[g]}\right) \oplus \mathcal{V}_{[g]}
$$

Lemma 1. For any $g \in \Sigma$ and $h, k \in[g]$ we have $\left\{\mathcal{P}_{h}, \mathcal{P}_{k}\right\}+\mathcal{P}_{h} \mathcal{P}_{k} \subset \mathcal{I}_{[g]}$. 
Proof. If $\left\{\mathcal{P}_{h}, \mathcal{P}_{k}\right\} \neq 0$ we have two possibilities. In the first one $h+k+g_{0} \in\left\{0, g_{0},-g_{0}\right\}$ and then $\left\{\mathcal{P}_{h}, \mathcal{P}_{k}\right\} \subset \mathcal{P}_{h+k+g_{0},[g]}$, or $h+k+g_{0} \notin\left\{0, g_{0},-g_{0}\right\}$ being then $\left\{h \otimes 0, k \otimes g_{0}\right\}$ a connection from $h$ to $h+k+g_{0}$, that is, $h+k+g_{0} \in[g]$ and so $\left\{\mathcal{P}_{h}, \mathcal{P}_{k}\right\} \subset \mathcal{I}_{[g]}$.

If $\mathcal{P}_{h} \mathcal{P}_{k} \neq 0$, we also have two cases to distinguish. In the first one $h+k \in\left\{0, g_{0},-g_{0}\right\}$ and so $\mathcal{P}_{h} \mathcal{P}_{k} \subset$ $\mathcal{P}_{h+k,[g]}$, while in the second one $h+k \notin\left\{0, g_{0},-g_{0}\right\}$ and then the connection $\{h \otimes 0, k \otimes 0\}$ gives us $h$ is connected to $h+k$ being $h+k \in[g]$. Consequently $\mathcal{P}_{h} \mathcal{P}_{k} \subset \mathcal{I}_{[g]}$.

Definition 6. For any $\alpha \in\left\{ \pm n g_{0}: n \in 0,1,2,3\right\}$ it is said that $\mathcal{P}_{\alpha}$ is tight if

$$
\begin{gathered}
\mathcal{P}_{\alpha}= \\
\sum_{\left\{h, p \in \Sigma \backslash\left\{ \pm n g_{0}: n \in 2,3\right\}: p=-h-g_{0}+\alpha\right\}}\left\{\mathcal{P}_{h}, \mathcal{P}_{p}\right\}+\sum_{\left\{k, q \in \Sigma \backslash\left\{ \pm n g_{0}: n \in 2,3\right\}: q=-k+\alpha\right\}} \mathcal{P}_{k} \mathcal{P}_{q} .
\end{gathered}
$$

Lemma 2. If $\mathcal{P}_{g_{0}}$ is tight then the following assertions hold.

1. If $-2 g_{0} \in \Sigma$ then $\left\{\mathcal{P}_{-2 g_{0}}, \mathcal{P}_{g_{0}}\right\} \subset \mathcal{P}_{0,\left[-2 g_{0}\right]}$ and $\mathcal{P}_{-2 g_{0}} \mathcal{P}_{g_{0}} \subset \mathcal{P}_{-g_{0},\left[-2 g_{0}\right]}$.

2. If $-3 g_{0} \in \Sigma$ then $\left\{\mathcal{P}_{-3 g_{0}}, \mathcal{P}_{g_{0}}\right\} \subset \mathcal{P}_{-g_{0},\left[-3 g_{0}\right]}$.

3. If $-2 g_{0}, g \in \Sigma$ with $\left\{\mathcal{P}_{-2 g_{0}}, \mathcal{P}_{0,[g]}\right\} \neq 0$ and $G$ is free of 2 -torsion, then $[g]=\left[-2 g_{0}\right]$ and

$$
\left\{\mathcal{P}_{-2 g_{0}}, \mathcal{P}_{0,[g]}\right\} \subset \mathcal{P}_{-g_{0},\left[-2 g_{0}\right]} \text {. }
$$

Proof. 1. Let us begin by showing $\left\{\mathcal{P}_{-2 g_{0}}, \mathcal{P}_{g_{0}}\right\} \subset \mathcal{P}_{0,\left[-2 g_{0}\right]}$. By Jacobi identity, Leibniz identity and anticommutativity

$$
\begin{aligned}
& \left\{\mathcal{P}_{-2 g_{0}}, \mathcal{P}_{g_{0}}\right\} \subset \\
& \sum_{h \in \Sigma \backslash\left\{ \pm n g_{0}: n \in 2,3\right\}}\left\{\mathcal{P}_{-2 g_{0}},\left\{\mathcal{P}_{h}, \mathcal{P}_{-h}\right\}\right\}+\sum_{k,-k+g_{0} \in \Sigma \backslash\left\{ \pm n g_{0}: n \in 2,3\right\}}\left\{\mathcal{P}_{-2 g_{0}}, \mathcal{P}_{k} \mathcal{P}_{-k+g_{0}}\right\} \\
& \subset \sum_{h \in \Sigma \backslash\left\{ \pm n g_{0}: n \in 2,3\right\}}\left(\left\{\mathcal{P}_{h-g_{0}}, \mathcal{P}_{-h}\right\}+\left\{\mathcal{P}_{-h-g_{0}}, \mathcal{P}_{h}\right\}\right)+ \\
& \sum_{k,-k+g_{0} \in \Sigma \backslash\left\{ \pm n g_{0}: n \in 2,3\right\}}\left(\mathcal{P}_{k-g_{0}} \mathcal{P}_{-k+g_{0}}+\mathcal{P}_{k}\left\{\mathcal{P}_{-2 g_{0}}, \mathcal{P}_{-k+g_{0}}\right\}\right) .
\end{aligned}
$$

Since for any $p \in \Sigma$ such that $\epsilon p+\nu g_{0} \notin\left\{0, g_{0},-g_{0}\right\}$, where $\epsilon, \nu \in\{ \pm 1\}$, we have $\epsilon p+\nu g_{0} \in \Sigma$ in case $\mathcal{P}_{\epsilon p+\nu g_{0}} \neq 0$, and the connection $\left\{-2 g_{0} \otimes 0, \epsilon p \otimes g_{0}\right\}$ gives us that in case $\mathcal{P}_{\epsilon p-g_{0}} \neq 0$ then $\epsilon p-g_{0} \in\left[-2 g_{0}\right]$ for any $\epsilon \in\{ \pm 1\}$, we get that any

$$
\left\{\mathcal{P}_{h-g_{0}}, \mathcal{P}_{-h}\right\}+\left\{\mathcal{P}_{-h-g_{0}}, \mathcal{P}_{h}\right\}+\mathcal{P}_{k-g_{0}} \mathcal{P}_{-k+g_{0}} \subset \mathcal{P}_{0,\left[-2 g_{0}\right]}
$$

Finally, observe that if $\left\{\mathcal{P}_{-2 g_{0}}, \mathcal{P}_{-k+g_{0}}\right\} \neq 0$ then $\left\{-2 g_{0} \otimes 0,\left(-k+g_{0}\right) \otimes g_{0}\right\}$ is a connection from $-2 g_{0}$ to $k$ and so

$$
\mathcal{P}_{k}\left\{\mathcal{P}_{-2 g_{0}}, \mathcal{P}_{-k+g_{0}}\right\} \subset \mathcal{P}_{k} \mathcal{P}_{-k} \subset \mathcal{P}_{0,\left[-2 g_{0}\right]}
$$

From Equations (2), (3) and (4) we complete the assertion.

Let us now prove that $\mathcal{P}_{-2 g_{0}} \mathcal{P}_{g_{0}} \subset \mathcal{P}_{-g_{0},\left[-2 g_{0}\right]}$. By Leibniz identity and associativity we get

$$
\begin{gathered}
\sum_{h \in \Sigma \backslash\left\{ \pm n g_{0}: n \in 2,3\right\}} \mathcal{P}_{-2 g_{0}}\left\{\mathcal{P}_{h}, \mathcal{P}_{-h}\right\}+\mathcal{P}_{k,-k+g_{0} \in \Sigma \backslash\left\{ \pm n g_{0}: n \in 2,3\right\}} \mathcal{P}_{g_{0}} \subset \mathcal{P}_{-2 g_{0}}\left(\mathcal{P}_{k} \mathcal{P}_{-k+g_{0}}\right) \subset \\
\sum_{h \in \Sigma \backslash\left\{ \pm n g_{0}: n \in 2,3\right\}}\left(\left\{\mathcal{P}_{h}, \mathcal{P}_{-h-2 g_{0}}\right\}+\mathcal{P}_{h-g_{0}} \mathcal{P}_{-h}\right)+\sum_{k,-k+g_{0} \in \Sigma \backslash\left\{ \pm n g_{0}: n \in 2,3\right\}} \mathcal{P}_{k-2 g_{0}} \mathcal{P}_{-k+g_{0}} .
\end{gathered}
$$

Now observe that for any $p \in \Sigma$ such that $\epsilon p-2 g_{0} \in \Sigma$, where $\epsilon \in\{ \pm 1\}$, the connection $\left\{\epsilon p-2 g_{0} \otimes 0,-\epsilon p \otimes 0\right\}$ gives us $\epsilon p-2 g_{0} \in\left[-2 g_{0}\right]$. From here, in case $-p+g_{0} \in \Sigma$, we get $\left\{\mathcal{P}_{p}, \mathcal{P}_{-p-2 g_{0}}\right\}+\mathcal{P}_{p-2 g_{0}} \mathcal{P}_{-p+g_{0}} \subset$ $\mathcal{P}_{-g_{0},\left[-2 g_{0}\right]}$. Finally, in case $p-g_{0} \in \Sigma$ we have seen above that $p-g_{0} \in\left[-2 g_{0}\right]$ and then $\mathcal{P}_{p-g_{0}} \mathcal{P}_{-p} \subset$ $\mathcal{P}_{-g_{0},\left[-2 g_{0}\right]}$ which completes this case.

2. Analogous to the first part of item 1.

3. We have

$$
0 \neq\left\{\mathcal{P}_{-2 g_{0}}, \mathcal{P}_{0,[g]}\right\} \subset\left\{\mathcal{P}_{-2 g_{0}}, \sum_{\left\{h \in[g]:-h-g_{0} \in \Sigma\right\}}\left\{\mathcal{P}_{h}, \mathcal{P}_{-h-g_{0}}\right\}+\sum_{k \in[g]} \mathcal{P}_{k} \mathcal{P}_{-k}\right\} \subset
$$




$$
\sum_{\left\{h \in[g]:-h-g_{0} \in \Sigma\right\}}\left(\left\{\mathcal{P}_{h-g_{0}}, \mathcal{P}_{-h-g_{0}}\right\}+\left\{\mathcal{P}_{-h-2 g_{0}}, \mathcal{P}_{h}\right\}\right)+\sum_{k \in[g]}\left(\mathcal{P}_{k-g_{0}} \mathcal{P}_{-k}+\mathcal{P}_{k} \mathcal{P}_{-k-g_{0}}\right) .
$$

If $\left\{\mathcal{P}_{h-g_{0}}, \mathcal{P}_{-h-g_{0}}\right\} \neq 0$ for some $h \in[g]$ with $-h-g_{0} \in \Sigma$, then the connection $\left\{h \otimes 0, g_{0} \otimes 0,-h \otimes g_{0}\right\}$ gives us $[h]=\left[-2 g_{0}\right]$ and so $[g]=\left[-2 g_{0}\right]$. Since $-h-g_{0} \in[h]=\left[-2 g_{0}\right]$ we also have $\left\{\mathcal{P}_{h-g_{0}}, \mathcal{P}_{-h-g_{0}}\right\} \subset$ $\mathcal{P}_{-g_{0},\left[-2 g_{0}\right]}$ when $h-g_{0} \notin\left\{0, g_{0},-g_{0}\right\}$, that is, when $h \neq 2 g_{0}$. In case $h=2 g_{0}$ we are dealing with the product $0 \neq\left\{\mathcal{P}_{g_{0}}, \mathcal{P}_{-3 g_{0}}\right\}$, but the facts $G$ is free of 2 -torsion and $2 g_{0} \in \Sigma$ show $-3 g_{0} \in \Sigma$ and so by item 2 we get $0 \neq\left\{\mathcal{P}_{g_{0}}, \mathcal{P}_{-3 g_{0}}\right\} \subset \mathcal{P}_{-g_{0},\left[-3 g_{0}\right]}=\mathcal{P}_{-g_{0},\left[-2 g_{0}\right]}$, where last equality is consequence of Remark1]

If $\mathcal{P}_{k} \mathcal{P}_{-k-g_{0}} \neq 0$ for some $k \in[g]$ we have as in the previous case that if $-k-g_{0} \in \Sigma$ then $[g]=[k]=$ $\left[-2 g_{0}\right]$, and consequently $\mathcal{P}_{k} \mathcal{P}_{-k-g_{0}} \subset \mathcal{P}_{-g_{0},\left[-2 g_{0}\right]}$. If $-k-g_{0} \in\left\{0, g_{0},-g_{0}\right\}$, then $k=-2 g_{0}$ and so $[g]=$ $[k]=\left[-2 g_{0}\right]$ and we are dealing with the product $0 \neq \mathcal{P}_{-2 g_{0}} \mathcal{P}_{g_{0}}$. But by Item $1,0 \neq \mathcal{P}_{-2 g_{0}} \mathcal{P}_{g_{0}} \subset \mathcal{P}_{-g_{0},\left[-2 g_{0}\right]}$.

If $\left\{\mathcal{P}_{-h-2 g_{0}}, \mathcal{P}_{h}\right\} \neq 0$ then in case $-h-2 g_{0} \in \Sigma$ we get $[g]=[h]=\left[-h-2 g_{0}\right]$ by Remark 1 . We observe that $-h-2 g_{0} \in \Sigma$. Indeed, in the opposite case $-h-2 g_{0} \in\left\{0, g_{0},-g_{0}\right\}$ and so $h=-2 g_{0}$, but then $-h-2 g_{0}=0$ being $-h-2 g_{0} \in \Sigma$, a contradiction. From here, the connection $\left\{-h-2 g_{0} \otimes 0, h \otimes 0\right\}$ shows $[g]=[h]=\left[-2 g_{0}\right]$ and consequently $0 \neq\left\{\mathcal{P}_{-h-2 g_{0}}, \mathcal{P}_{h}\right\} \subset \mathcal{P}_{-g_{0},\left[-2 g_{0}\right]}$.

Finally, if $\mathcal{P}_{k-g_{0}} \mathcal{P}_{-k} \neq 0$, we have that in case $k-g_{0} \in \Sigma$ then $\left[k-g_{0}\right]=[k]=[g]$ by Remark 1 and that the connection $\left\{k-g_{0} \otimes 0,-k \otimes-g_{0}\right\}$ shows $\left[k-g_{0}\right]=\left[-2 g_{0}\right]$. Consequently $[g]=\left[2 g_{0}\right]$ and $0 \neq \mathcal{P}_{k-g_{0}} \mathcal{P}_{-k} \subset \mathcal{P}_{-g_{0},\left[-2 g_{0}\right]}$. If $k-g_{0} \notin \Sigma$ then $k=2 g_{0}$ and we are dealing with the product $0 \neq \mathcal{P}_{g_{0}} \mathcal{P}_{-2 g_{0}}$ which is contained in $\mathcal{P}_{-g_{0},\left[-2 g_{0}\right]}$ by Item 1 .

Lemma 3. Suppose $G$ is free of 2-torsion, then for any $\alpha, \beta \in\left\{0, g_{0},-g_{0}\right\}$ the following assertions hold.

1. For each $h \in \Sigma$ satisfying $-h-g_{0}+\alpha \in \Sigma$ we have

1.1. if $\alpha+\beta+g_{0} \in\left\{0, g_{0},-g_{0}\right\}$ then $h+\beta+g_{0} \in \Sigma$ in case $\mathcal{P}_{h+\beta+g_{0}} \neq 0$, and $-h+\alpha+\beta \in \Sigma$ in case $\mathcal{P}_{-h+\alpha+\beta} \neq 0$.

1.2. if $\alpha+\beta \in\left\{0, g_{0},-g_{0}\right\}$ and $\mathcal{P}_{h+\beta+g_{0}} \neq 0$ then $h+\beta+g_{0} \in \Sigma \cup\left\{-g_{0}\right\}$.

1.3. if $\alpha+\beta \in\left\{0, g_{0},-g_{0}\right\}$ and $(\alpha, \beta, h) \notin\left\{\left(g_{0},-g_{0},-2 g_{0}\right),\left(0,-g_{0},-3 g_{0}\right)\right\}$ then $-h-g_{0}+\alpha+\beta \in$ $\Sigma$ in case $\mathcal{P}_{-h-g_{0}+\alpha+\beta} \neq 0$.

2. For each $k \in \Sigma$ satisfying $-k+\alpha \in \Sigma \cup\left\{-g_{0}\right\}$ we have

2.1. if $\alpha+\beta+g_{0} \in\left\{0, g_{0},-g_{0}\right\}$ and $\mathcal{P}_{k+\beta+g_{0}} \neq 0$, then $k+\beta+g_{0} \in \Sigma \cup\left\{-g_{0}\right\}$.

2.2. if $\alpha+\beta+g_{0} \in\left\{0, g_{0},-g_{0}\right\}$ and $\mathcal{P}_{-k+\alpha} \neq 0$, then $-k+\alpha \in \Sigma$.

2.3. if $\alpha+\beta \in\left\{0, g_{0},-g_{0}\right\}$ and $\mathcal{P}_{-k+\alpha+\beta} \neq 0$, then $-k+\alpha+\beta \in \Sigma \cup\left\{-g_{0}\right\}$.

Proof. 1.1. Suppose $\mathcal{P}_{h+\beta+g_{0}} \neq 0$ and $h+\beta+g_{0} \notin \Sigma$ being then $h+\beta+g_{0} \in\left\{0, g_{0},-g_{0}\right\}$.

If $h+\beta+g_{0}=0$, as $\beta \in\left\{0, g_{0},-g_{0}\right\}$ and $h \in \Sigma$, then necessarily $\beta=g_{0}$ and $h=-2 g_{0}$ with

$$
2 g_{0} \notin\left\{0, g_{0},-g_{0}\right\} .
$$

Since $-h-g_{0}+\alpha \in \Sigma$ then $g_{0}+\alpha \in \Sigma$ and, taking into account $\alpha \in\left\{0, g_{0},-g_{0}\right\}$, we get $\alpha=g_{0}$. But we also know $\alpha+\beta+g_{0} \in\left\{0, g_{0},-g_{0}\right\}$ and so $3 g_{0} \in\left\{0, g_{0},-g_{0}\right\}$. This implies either $2 g_{0} \in\left\{-g_{0}, 0\right\}$ or $4 g_{0}=0$. In the first case we have a contradiction with Equation (5) while in the second one $0 \neq 2 g_{0}$ is an element of $G$ with 2-torsion which is also a contradiction. A similar argument gives us that the case $h+\beta+g_{0} \in \pm g_{0}$ does not hold and so $h+\beta+g_{0} \in \Sigma$. We can also show as above that $-h+\alpha+\beta \in \Sigma$ in case $\mathcal{P}_{-h+\alpha+\beta} \neq 0$.

The remaining items can be proved by arguing as in Item 1.1.

Lemma 4. Suppose $G$ is free of 2-torsion, then for any $g \in \Sigma$ and $\alpha, \beta \in\left\{0, g_{0},-g_{0}\right\}$ we have

1. $\left\{\mathcal{P}_{\alpha,[g]}, \mathcal{P}_{\beta,[g]}\right\} \subset \mathcal{I}_{[g]}$.

2. If furthermore $\mathcal{P}_{g_{0}}$ is tight then $\mathcal{P}_{\alpha,[g]} \mathcal{P}_{\beta,[g]} \subset \mathcal{I}_{[g]}$.

Proof. 1. Suppose there exists $h \in[g]$ with $-h-g_{0}+\alpha \in \Sigma$ such that

$$
0 \neq\left\{\left\{\mathcal{P}_{h}, \mathcal{P}_{-h-g_{0}+\alpha}\right\}, \mathcal{P}_{\beta,[g]}\right\} \subset \mathcal{P}_{\alpha+\beta+g_{0}},
$$

or there is $k \in[g]$ with $-k+\alpha \in \Sigma \cup\left\{-g_{0}\right\}$ satisfying

$$
0 \neq\left\{\mathcal{P}_{k} \mathcal{P}_{-k+\alpha}, \mathcal{P}_{\beta,[g]}\right\} \subset \mathcal{P}_{\alpha+\beta+g_{0}} .
$$

Let us study Equation (6), by applying Jacobi identity and anticommutativity we get

$$
\begin{gathered}
0 \neq\left\{\left\{\mathcal{P}_{h}, \mathcal{P}_{-h-g_{0}+\alpha}\right\}, \mathcal{P}_{\beta}\right\} \subset \\
\left\{\mathcal{P}_{h+\beta+g_{0}}, \mathcal{P}_{-h-g_{0}+\alpha}\right\}+\left\{\mathcal{P}_{h}, \mathcal{P}_{-h+\alpha+\beta}\right\} .
\end{gathered}
$$


We are going to distinguish two possibilities, in the first one

$$
\alpha+\beta+g_{0} \in\left\{0, g_{0},-g_{0}\right\} .
$$

From Equation (8), either $\left\{\mathcal{P}_{h+\beta+g_{0}}, \mathcal{P}_{-h-g_{0}+\alpha}\right\} \neq 0$ or $\left\{\mathcal{P}_{h}, \mathcal{P}_{-h+\alpha+\beta}\right\} \neq 0$. In the first case, Lemma 3 -1.1 gives us $h+\beta+g_{0} \in \Sigma$. Hence, taking into account that $\mathcal{P}_{\beta,[g]} \subset \mathcal{P}_{\beta}$, that the connection $\left\{h \otimes 0, \beta \otimes g_{0}\right\}$ shows


a similar way we have that in case $\left\{\mathcal{P}_{h}, \mathcal{P}_{-h+\alpha+\beta}\right\} \neq 0$ then $0 \neq\left\{\mathcal{P}_{h}, \mathcal{P}_{-h+\alpha+\beta}\right\} \subset \mathcal{P}_{\alpha+\beta+g_{0},[g]}$ and so we can assert

In the second possibility

$$
\left\{\left\{\mathcal{P}_{h}, \mathcal{P}_{-h-g_{0}+\alpha}\right\}, \mathcal{P}_{\beta,[g]}\right\} \subset \mathcal{P}_{\alpha+\beta+g_{0},[g]}
$$

$$
\alpha+\beta+g_{0} \notin\left\{0, g_{0},-g_{0}\right\} .
$$

We also have from Equation (8) that either $\left\{\mathcal{P}_{h+\beta+g_{0}}, \mathcal{P}_{-h-g_{0}+\alpha}\right\} \neq 0$ or $\left\{\mathcal{P}_{h}, \mathcal{P}_{-h+\alpha+\beta}\right\} \neq 0$. In the first case, since $-h-g_{0}+\alpha \in \Sigma$, the connection

$$
\left\{h \otimes 0, g_{0} \otimes-\alpha,\left(-h-\beta-g_{0}\right) \otimes-g_{0}\right\}
$$

gives us $\alpha+\beta+g_{0} \in[h]=[g]$ while in the second one the connection

$$
\left\{h \otimes 0,(-h+\alpha+\beta) \otimes g_{0}\right\}
$$

gives us also $\alpha+\beta+g_{0} \in[h]=[g]$. We have shown $\left\{\left\{\mathcal{P}_{h}, \mathcal{P}_{-h-g_{0}+\alpha}\right\}, \mathcal{P}_{\beta,[g]}\right\} \subset \mathcal{V}_{[g]}$ in this case and taking also into account Equation (9] that

$$
\left\{\left\{\mathcal{P}_{h}, \mathcal{P}_{-h-g_{0}+\alpha}\right\}, \mathcal{P}_{\beta,[g]}\right\} \subset \mathcal{I}_{[g]} .
$$

From Leibniz identity and anticommutativity we can study Equation (7) in a similar way to the above study of Equation (6), taking now into account Lemma3-2.1. and 2.2. to get

$$
\left\{\mathcal{P}_{k} \mathcal{P}_{-k+\alpha}, \mathcal{P}_{\beta,[g]}\right\} \subset \mathcal{I}_{[g]} .
$$

and so we conclude

$$
\left\{\mathcal{P}_{\alpha,[g]}, \mathcal{P}_{\beta,[g]}\right\} \subset \mathcal{I}_{[g]} .
$$

2. Suppose there exists $h \in[g]$ satisfying $-h-g_{0}+\alpha \in \Sigma$ and such that

$$
0 \neq\left\{\mathcal{P}_{h}, \mathcal{P}_{-h-g_{0}+\alpha}\right\} \mathcal{P}_{\beta,[g]} \subset \mathcal{P}_{\alpha+\beta},
$$

or there is $k \in[g]$ with $-k+\alpha \in \Sigma \cup\left\{-g_{0}\right\}$ such that

$$
0 \neq\left(\mathcal{P}_{k} \mathcal{P}_{-k+\alpha}\right) \mathcal{P}_{\beta,[g]} \subset \mathcal{P}_{\alpha+\beta}
$$

An analogous argument to item 1, taking now into account Lemma 3-1.2., 1.3. and 2.3., and also in the first possibility that the fact $\mathcal{P}_{g_{0}}$ tight together with Remark 1 imply that in case $-2 g_{0} \in[g]$ then $\left\{\mathcal{P}_{-2 g_{0}}, \mathcal{P}_{g_{0}}\right\} \in$ $\mathcal{P}_{0,[g]}$ and that in case $-3 g_{0} \in[g]$ then $\left\{\mathcal{P}_{-3 g_{0}}, \mathcal{P}_{g_{0}}\right\} \in \mathcal{P}_{-g_{0},[g]}$, gives us

$$
\left\{\mathcal{P}_{h}, \mathcal{P}_{-h-g_{0}+\alpha}\right\} \mathcal{P}_{\beta,[g]}+\left(\mathcal{P}_{k} \mathcal{P}_{-k+\alpha}\right) \mathcal{P}_{\beta,[g]} \subset \mathcal{I}_{[g]}
$$

and so

$$
\mathcal{P}_{\alpha,[g]} \mathcal{P}_{\beta,[g]} \subset \mathcal{I}_{[g]}
$$

Lemma 5. Suppose $\mathcal{P}_{g_{0}}$ is tight and $G$ is free of 2-torsion, then for any $g \in \Sigma, \alpha \in\left\{0, g_{0},-g_{0}\right\}$ and $k \in[g]$ we have

$$
\left\{\mathcal{P}_{\alpha,[g]}, \mathcal{P}_{k}\right\}+\mathcal{P}_{\alpha,[g]} \mathcal{P}_{k} \subset \mathcal{I}_{[g]}
$$

Proof. Suppose $\left\{\mathcal{P}_{\alpha,[g]}, \mathcal{P}_{k}\right\} \neq 0$. We have two cases to distinguish. In the first one $\alpha+k+g_{0} \notin\left\{0, g_{0},-g_{0}\right\}$ and so $\alpha+k+g_{0} \in \Sigma$. Then we have that the connection $\left\{k \otimes 0, \alpha \otimes g_{0}\right\}$ gives us $\alpha+k+g_{0} \in[k]=[g]$ and SO

$$
\left\{\mathcal{P}_{\alpha,[g]}, \mathcal{P}_{k}\right\} \subset \mathcal{V}_{[g]}
$$

In the second case $\alpha+k+g_{0} \in\left\{0, g_{0},-g_{0}\right\}$. Taking also into account $\alpha \in\left\{0, g_{0},-g_{0}\right\}$ and $k \notin\left\{0, g_{0},-g_{0}\right\}$ we have that

Consider the possibility $(\alpha, k)=\left(g_{0},-2 g_{0}\right)$, that is,

$$
(\alpha, k) \in\left\{\left(g_{0},-2 g_{0}\right),\left(0,-2 g_{0}\right),\left(g_{0},-3 g_{0}\right)\right\} .
$$

$$
0 \neq\left\{\mathcal{P}_{g_{0},[g]}, \mathcal{P}_{-2 g_{0}}\right\} \subset \mathcal{P}_{0}
$$


being $-2 g_{0}=k \in \Sigma$. From here, $\left[-2 g_{0}\right]=[g]$ and by Lemma2 1 we get

$$
0 \neq\left\{\mathcal{P}_{g_{0},[g]}, \mathcal{P}_{-2 g_{0}}\right\} \subset\left\{\mathcal{P}_{g_{0}}, \mathcal{P}_{-2 g_{0}}\right\} \subset \mathcal{P}_{0,\left[-2 g_{0}\right]}=\mathcal{P}_{0,\left[g_{0}\right]} .
$$

In a similar way we can show $0 \neq\left\{\mathcal{P}_{\alpha,[g]}, \mathcal{P}_{k}\right\} \subset \mathcal{P}_{\alpha+g_{0}+k,[g]}$ when $(\alpha, k) \in\left\{\left(0,-2 g_{0}\right),\left(g_{0},-3 g_{0}\right)\right\}$ and we conclude

$$
\left\{\mathcal{P}_{\alpha,[g]}, \mathcal{P}_{k}\right\} \subset \mathcal{I}_{[g]} .
$$

Suppose now $\mathcal{P}_{\alpha,[g]} \mathcal{P}_{k} \neq 0$. If $\alpha+k \notin\left\{0, g_{0},-g_{0}\right\}$ then the connection $\{k \otimes 0, \alpha \otimes 0\}$ gives us

$$
0 \neq \mathcal{P}_{\alpha,[g]} \mathcal{P}_{k} \subset \mathcal{P}_{k+\alpha} \subset \mathcal{V}_{[g]} .
$$

If $\alpha+k \in\left\{0, g_{0},-g_{0}\right\}$ then $(\alpha, k) \in\left\{\left(g_{0},-2 g_{0}\right),\left(-g_{0}, 2 g_{0}\right)\right\}$. If $(\alpha, k)=\left(g_{0},-2 g_{0}\right)$ then $0 \neq$ $\mathcal{P}_{g_{0},[g]} \mathcal{P}_{-2 g_{0}} \subset \mathcal{P}_{g_{0}} \mathcal{P}_{-2 g_{0}} \subset \mathcal{P}_{-g_{0},[g]}$, last inclusion being consequence of Lemma 2 - 1 and $\left[-2 g_{0}\right]=[g]$. Finally, if $(\alpha, k)=\left(-g_{0}, 2 g_{0}\right)$ then $0 \neq \mathcal{P}_{-g_{0},[g]} \mathcal{P}_{2 g_{0}} \subset \mathcal{P}_{-g_{0}} \mathcal{P}_{2 g_{0}}=\mathcal{P}_{2 g_{0}} \mathcal{P}_{-g_{0}} \subset \mathcal{P}_{-g_{0},\left[2 g_{0}\right]}=\mathcal{P}_{-g_{0},[g]}$. We have shown

$$
\mathcal{P}_{\alpha,[g]} \mathcal{P}_{k} \subset \mathcal{I}_{[g]} .
$$

Proposition 2. Suppose $\mathcal{P}_{g_{0}}$ is tight and $G$ is free of 2-torsion, then for any $g \in \Sigma$ the graded linear subspace $\mathcal{I}_{[g]}$ is a subalgebra of $\mathcal{P}$.

Proof. Since $\mathcal{I}_{[g]}=\left(\sum_{\alpha \in\left\{0, g_{0},-g_{0}\right\}} \mathcal{P}_{\alpha,[g]}\right) \oplus \mathcal{V}_{[g]}$ we can write

$$
\begin{aligned}
& \left\{\mathcal{I}_{[g]}, \mathcal{I}_{[g]}\right\} \subset \\
& \sum_{\alpha, \beta \in\left\{0, g_{0},-g_{0}\right\}}\left\{\mathcal{P}_{\alpha,[g]}, \mathcal{P}_{\beta,[g]}\right\}+\sum_{\alpha \in\left\{0, g_{0},-g_{0}\right\}}\left\{\mathcal{P}_{\alpha,[g]}, \mathcal{V}_{[g]}\right\}+\left\{\mathcal{V}_{[g]}, \mathcal{V}_{[g]}\right\} .
\end{aligned}
$$

From here, Lemmas 1,4 and 5 allow us to get $\left\{\mathcal{I}_{[g]}, \mathcal{I}_{[g]}\right\} \subset \mathcal{I}_{[g]}$.

In a similar way we have $\mathcal{I}_{[g]} \mathcal{I}_{[g]} \subset \mathcal{I}_{[g]}$ and consequently $\mathcal{I}_{[g]}$ is a subalgebra of $\mathcal{P}$.

We call $\mathcal{I}_{[g]}$ the subalgebra of $\mathcal{P}$ associated to $[g]$.

\section{DeCOMPOSITIONS AS SUM OF IDEALS}

We begin this section by showing that for any $g \in \Sigma$, the subalgebra $\mathcal{I}_{[g]}$ is actually an ideal of $\mathcal{P}$. From now on the group $G$ will be suppose free of 2-torsion.

Proposition 3. If $[g] \neq[h]$ for some $g, h \in \Sigma$ then $\left\{\mathcal{I}_{[g]}, \mathcal{I}_{[h]}\right\}+\mathcal{I}_{[g]} \mathcal{I}_{[h]}=0$.

Proof. We have to study the products

$$
\begin{gathered}
\left\{\mathcal{I}_{[g]}, \mathcal{I}_{[h]}\right\}= \\
\left\{\left(\sum_{\alpha \in\left\{0, g_{0},-g_{0}\right\}} \mathcal{P}_{\alpha,[g]}\right) \oplus \mathcal{V}_{[g]},\left(\sum_{\alpha \in\left\{0, g_{0},-g_{0}\right\}} \mathcal{P}_{\alpha,[h]}\right) \oplus \mathcal{V}_{[h]}\right\}
\end{gathered}
$$

and

$$
\left(\left(\sum_{\alpha \in\left\{0, g_{0},-g_{0}\right\}} \mathcal{I}_{[g]} \mathcal{I}_{[h]}=\mathcal{V}_{[g]}\right)\left(\left(\sum_{\alpha \in\left\{0, g_{0},-g_{0}\right\}} \mathcal{P}_{\alpha,[h]}\right) \oplus \mathcal{V}_{[h]}\right) .\right.
$$

We begin by considering the summand $\left\{\mathcal{V}_{[g]}, \mathcal{V}_{[h]}\right\}$ of the first product. Suppose there exist $k \in[g]$ and $l \in[h]$ such that $0 \neq\left\{\mathcal{P}_{k}, \mathcal{P}_{l}\right\} \subset \mathcal{P}_{k+l+g_{0}}$. We have to distinguish two cases. In the first one $k+l+g_{0} \notin\left\{0, g_{0},-g_{0}\right\}$ and so $k+l+g_{0} \in \Sigma$. Then the connection $\left\{k \otimes 0, l \otimes g_{0},-k \otimes-g_{0}\right\}$ gives us $[g]=[k]=[l]=[h]$, a contradiction. Hence $\left\{\mathcal{V}_{[g]}, \mathcal{V}_{[h]}\right\}=0$ in this case. In the second possibility, $k+l+g_{0} \in\left\{0, g_{0},-g_{0}\right\}$. From here $l \in\left\{-k,-g_{0}-k,-2 g_{0}-k\right\}$ and by Remark 1 we get $[k]=[l]$, a contradiction, then

$$
\left\{\mathcal{V}_{[g]}, \mathcal{V}_{[h]}\right\}=0
$$

in any case.

Consider now the summand $\mathcal{V}_{[g]} \mathcal{V}_{[h]}$ in the second product and suppose there exist $k \in[g]$ and $l \in[h]$ such that $0 \neq \mathcal{P}_{k} \mathcal{P}_{l} \subset \mathcal{P}_{k+l}$. In case $k+l \notin\left\{0, g_{0},-g_{0}\right\}$, the connection $\{k \otimes 0, l \otimes 0,-k \otimes 0\}$ gives us $[k]=[l]$, a 
contradiction, while in case $k+l \in\left\{0, g_{0},-g_{0}\right\}$ necessarily $l \in\left\{-k, g_{0}-k,-g_{0}-k\right\}$ being then, see Remark 1. $[k]=[l]$, a contradiction. We have shown

$$
\mathcal{V}_{[g]} \mathcal{V}_{[h]}=0
$$

In order to study the product $\left\{\sum_{\alpha \in\left\{0, g_{0},-g_{0}\right\}} \mathcal{P}_{\alpha,[g]}, \mathcal{V}_{[h]}\right\}$, consider any

$$
\left\{\left\{\mathcal{P}_{k}, \mathcal{P}_{-k-g_{0}+\alpha}\right\}, \mathcal{P}_{l}\right\}
$$

with $k \in[g]$ satisfying $-k-g_{0}+\alpha \in \Sigma$ and $l \in[h]$. We have by Jacobi identity and anticommutativity that

$$
\left\{\left\{\mathcal{P}_{k}, \mathcal{P}_{-k-g_{0}+\alpha}\right\}, \mathcal{P}_{l}\right\} \subset\left\{\left\{\mathcal{P}_{k}, \mathcal{P}_{l}\right\}, \mathcal{P}_{-k-g_{0}+\alpha}\right\}+\left\{\left\{\mathcal{P}_{-k-g_{0}+\alpha}, \mathcal{P}_{l}\right\}, \mathcal{P}_{k}\right\}
$$

Since by Equation (10) and Remark 1 we get $\left\{\mathcal{P}_{k}, \mathcal{P}_{l}\right\}=\left\{\mathcal{P}_{-k-g_{0}+\alpha}, \mathcal{P}_{l}\right\}=0$ we obtain $\left\{\left\{\mathcal{P}_{k}, \mathcal{P}_{-k-g_{0}+\alpha}\right\}, \mathcal{P}_{l}\right\}=0$. If we now take any $\left\{\mathcal{P}_{k} \mathcal{P}_{-k+\alpha}, \mathcal{P}_{l}\right\}$ with $k \in[g]$ such that $-k+\alpha \in \Sigma \cup\left\{-g_{0}\right\}$ and $l \in[h]$ then we get by Leibniz identity and commutativity that $\left\{\mathcal{P}_{k} \mathcal{P}_{-k+\alpha}, \mathcal{P}_{l}\right\} \subset\left\{\mathcal{P}_{l} \mathcal{P}_{k}\right\} \mathcal{P}_{-k+\alpha}+$ $\mathcal{P}_{k}\left\{\mathcal{P}_{l}, \mathcal{P}_{-k+\alpha}\right\}$, but by Equation (10) we have $\left\{\mathcal{P}_{l} \mathcal{P}_{k}\right\}=\left\{\mathcal{P}_{l}, \mathcal{P}_{-k+\alpha}\right\}=0$ in case $-k+\alpha \in \Sigma$. If $-k+\alpha=-g_{0}$ then $\mathcal{P}_{k}\left\{\mathcal{P}_{l}, \mathcal{P}_{-k+\alpha}\right\} \subset \mathcal{P}_{k} \mathcal{P}_{l}=0$ by Equation (11) being so $\left\{\mathcal{P}_{k} \mathcal{P}_{-k+\alpha}, \mathcal{P}_{l}\right\}=0$ in any case. We have proved

$$
\left\{\sum_{\alpha \in\left\{0, g_{0},-g_{0}\right\}} \mathcal{P}_{\alpha,[g]}, \mathcal{V}_{[h]}\right\}+\left\{\mathcal{V}_{[g]}, \sum_{\alpha \in\left\{0, g_{0},-g_{0}\right\}} \mathcal{P}_{\alpha,[h]}\right\}=0
$$

In a similar way as above, taking now into account Leibniz identity, commutativity and associativity we get

$$
\left(\sum_{\alpha \in\left\{0, g_{0},-g_{0}\right\}} \mathcal{P}_{\alpha,[g]}\right) \mathcal{V}_{[h]}+\mathcal{V}_{[g]}\left(\sum_{\alpha \in\left\{0, g_{0},-g_{0}\right\}} \mathcal{P}_{\alpha,[h]}\right)=0
$$

Finally, let us consider the case $\sum_{\alpha, \beta \in\left\{0, g_{0},-g_{0}\right\}}\left\{\mathcal{P}_{\alpha,[g]}, \mathcal{P}_{\beta,[h]}\right\}$. By arguing as in the previous case, taking now into account Equation (12) and the fact (easy to prove) $\left\{\mathcal{P}_{\beta,[h]}, \mathcal{P}_{-g_{0}}\right\} \subset \mathcal{P}_{\beta,[h]}$, we get

$$
\left\{\sum_{\alpha \in\left\{0, g_{0},-g_{0}\right\}} \mathcal{P}_{\alpha,[g]}, \sum_{\alpha \in\left\{0, g_{0},-g_{0}\right\}} \mathcal{P}_{\alpha,[h]}\right\}=0 .
$$

In a similar way, by considering now Equations (12) and (13), we get

$$
\left(\sum_{\alpha \in\left\{0, g_{0},-g_{0}\right\}} \mathcal{P}_{\alpha,[g]}\right)\left(\sum_{\alpha \in\left\{0, g_{0},-g_{0}\right\}} \mathcal{P}_{\alpha,[h]}\right)=0 .
$$

From Equations (10), (12) and (14) we get that $\left\{\mathcal{I}_{[g]}, \mathcal{I}_{[h]}\right\}=0$ while from Equations (11), (13) and (15) that $\mathcal{I}_{[g]} \mathcal{I}_{[h]}=0$ which complete the proof.

Theorem 1. Suppose any $\mathcal{P}_{\alpha}, \alpha \in\left\{0, g_{0},-g_{0}\right\}$, is tight then the following assertions hold.

1. For any $g \in \Sigma$, the subalgebra $\mathcal{I}_{[g]}$ of $\mathcal{P}$ associated to $[g]$ is an ideal of $\mathcal{P}$.

2. If $\mathcal{P}$ is simple, then there exists a connection between any two elements of $\Sigma$.

Proof. 1. Since we can write $\bigoplus_{h \in \Sigma} \mathcal{P}_{h}=\bigoplus_{[h] \in \Sigma / \sim} \mathcal{V}_{[h]}$ and $\mathcal{P}_{\alpha}=\sum_{[h] \in \Sigma / \sim} \mathcal{P}_{\alpha,[h]}$ for any $\alpha \in\left\{0, g_{0},-g_{0}\right\}$,

we have

$$
\mathcal{P}=\mathcal{P}_{0}+\mathcal{P}_{g_{0}}+\mathcal{P}_{-g_{0}} \oplus\left(\bigoplus_{h \in \Sigma} \mathcal{P}_{h}\right)=\sum_{[h] \in \Sigma / \sim} \mathcal{I}_{[h]}
$$

From here, by Propositions 2 and 3 we have

$$
\left\{\mathcal{I}_{[g]}, \mathcal{P}\right\}+\left\{\mathcal{P}, \mathcal{I}_{[g]}\right\}+\mathcal{I}_{[g]} \mathcal{P}+\mathcal{P} \mathcal{I}_{[g]} \subset\left\{\mathcal{I}_{[g]}, \mathcal{I}_{[g]}\right\}+\sum_{[h] \neq[g]}\left\{\mathcal{I}_{[g]}, \mathcal{I}_{[h]}\right\}+\mathcal{I}_{[g]} \mathcal{I}_{[g]}+\sum_{[h] \neq[g]} \mathcal{I}_{[g]} \mathcal{I}_{[h]} \subset \mathcal{I}_{[g]}
$$

as desired.

(ii) The simplicity of $\mathcal{P}$ applies to get that $\mathcal{I}_{[g]}=\mathcal{P}$ for any $g \in \Sigma$. Hence $[g]=\Sigma$ and so any couple of elements in $\Sigma$ are connected.

As consequence of Equation (16, Theorem 1 and Proposition 3 we can state the following result. 
Theorem 2. Suppose any $\mathcal{P}_{\alpha}, \alpha \in\left\{0, g_{0},-g_{0}\right\}$, is tight. It follows

$$
\mathcal{P}=\sum_{[g] \in \Sigma / \sim} \mathcal{I}_{[g]}
$$

being any $\mathcal{I}_{[g]}$ one of the ideals given in Theorem 1 Moreover, $\left\{\mathcal{I}_{[g]}, \mathcal{I}_{[h]}\right\}+\mathcal{I}_{[g]} \mathcal{I}_{[h]}=0$ whenever $[g] \neq[h]$.

As usual, the center of $\mathcal{P}$ is defined as the set $\{v \in \mathcal{P}:\{v, \mathcal{P}\}+\{\mathcal{P}, v\}+v \mathcal{P}+\mathcal{P} v=0\}$.

Corollary 1. If $\mathcal{P}$ is centerless and any $\mathcal{P}_{\alpha}, \alpha \in\left\{0, g_{0},-g_{0}\right\}$, is tight then $\mathcal{P}$ is the direct sums of the ideals given in Theorem 1 ,

$$
\mathcal{P}=\bigoplus_{[g] \in \Sigma / \sim} \mathcal{I}_{[g]}
$$

Proof. We have to show the direct character of the sum. Given $x \in \mathcal{I}_{[g]} \cap \sum_{\substack{[h] \in \Sigma / \sim \\ h \nsim g}} \mathcal{I}_{[h]}$, by using the fact $\left\{\mathcal{I}_{[g]}, \mathcal{I}_{[h]}\right\}+\mathcal{I}_{[g]} \mathcal{I}_{[h]}=0$ for $[g] \neq[h]$ we obtain $\{x, \mathcal{P}\}+\{\mathcal{P}, x\}+x \mathcal{P}+\mathcal{P} x=0$. That is, $x$ belongs to the center of $\mathcal{P}$ and so $x=0$ as desired.

\section{THE SIMPLE COMPONENTS}

In this section we study if any of the components in the decomposition given in Corollary 1 is simple. Under mild conditions we give an affirmative answer and furthermore a second Wedderburn-type theorem is stated. Finally, we recall that in this section the group $G$ is supposed to be free of 2-torsion.

Lemma 6. Let $\mathcal{P}$ be centerless and with $\mathcal{P}_{\beta}$ tight for $\beta \in\left\{0, \pm g_{0}, \pm 2 g_{0},-3 g_{0}\right\}$. If $I$ is an ideal of $\mathcal{P}$ such that $I \subset \mathcal{P}_{0}+\mathcal{P}_{g_{0}}+\mathcal{P}_{-g_{0}}$ then $I=\{0\}$.

Proof. Suppose there exists a nonzero ideal $\mathcal{I}$ of $\mathcal{P}$ contained in $\mathcal{P}_{0}+\mathcal{P}_{g_{0}}+\mathcal{P}_{-g_{0}}$. Since can write

$$
I=\left(I \cap \mathcal{P}_{0}\right)+\left(I \cap \mathcal{P}_{g_{0}}\right)+\left(I \cap \mathcal{P}_{-g_{0}}\right),
$$

some $I \cap \mathcal{P}_{\alpha} \neq 0$ for $\alpha \in\left\{0, g_{0},-g_{0}\right\}$. Taking into account $\mathcal{P}$ is centerless, there exists $h \in \Sigma \cup\left\{0, g_{0},-g_{0}\right\}$ such that either $\left\{I \cap \mathcal{P}_{\alpha}, \mathcal{P}_{h}\right\} \neq 0$ or $\left(I \cap \mathcal{P}_{\alpha}\right) \mathcal{P}_{h} \neq 0$. In the first case, $0 \neq\left\{I \cap \mathcal{P}_{\alpha}, \mathcal{P}_{h}\right\} \subset \mathcal{P}_{\alpha+h+g_{0}} \cap\left(\mathcal{P}_{0}+\right.$ $\left.\mathcal{P}_{g_{0}}+\mathcal{P}_{-g_{0}}\right)$ and so necessarily

$$
h \in\left\{0, g_{0},-g_{0},-2 g_{0},-3 g_{0}\right\},
$$

but by tightness of the homogeneous spaces associated to these elements we have

$$
\begin{gathered}
0 \neq\left\{I \cap \mathcal{P}_{\alpha}, \mathcal{P}_{h}\right\} \subset\left\{I \cap \mathcal{P}_{\alpha}, \sum_{p,-p-g_{0}+h \in \Sigma \backslash\left\{ \pm n g_{0}: n \in 2,3\right\}}\left\{\mathcal{P}_{p}, \mathcal{P}_{-p-g_{0}+h}\right\}+\right. \\
\left\{I \cap \mathcal{P}_{\alpha}, \sum_{k,-k+h \in \Sigma \backslash\left\{ \pm n g_{0}: n \in 2,3\right\}} \mathcal{P}_{k} \mathcal{P}_{-k+h}\right\} .
\end{gathered}
$$

From here Jacobi identity and Leibniz identity give us that there exists some $r \notin\left\{0, g_{0},-g_{0},-2 g_{0},-3 g_{0}\right\}$ satisfying $\left\{I \cap \mathcal{P}_{\alpha}, \mathcal{P}_{r}\right\} \neq 0$ which contradicts Equation (17).

In the second case $0 \neq\left(I \cap \mathcal{P}_{\alpha}\right) \mathcal{P}_{h} \subset \mathcal{P}_{\alpha+h} \cap\left(\mathcal{P}_{0}+\mathcal{P}_{g_{0}}+\mathcal{P}_{-g_{0}}\right)$. This fact only occurs for

$$
h \in\left\{0, g_{0},-g_{0}, 2 g_{0},-2 g_{0}\right\} .
$$

A similar above argument with the tightness of the homogeneous spaces associated to these elements, Leibniz identity and associativity gives us a contradiction. Hence we conclude $I=0$.

Let us introduce the concepts of maximal length and $\Sigma$-multiplicativity in the setup of Poisson color algebras of degree $g_{0}$ in a similar way than in the frameworks of graded Lie algebras, graded Lie superalgebras, graded Leibniz algebras, split Poisson algebras, split color Lie algebras etc. (see [6, 7, 8, 9, 17] for discussion and examples on these concepts).

Definition 7. We say that a Poisson color algebra $\mathcal{P}$ of degree $g_{0}$ is of maximal length if $\mathcal{P}_{0} \neq 0$ and $\operatorname{dim} \mathcal{P}_{g}=1$ for any $g \in \Sigma$.

Definition 8. We say that a Poisson color algebra $\mathcal{P}$ of degree $g_{0}$ is $\Sigma$-multiplicative if given $g \in \Sigma$ and $h \in \Sigma \cup\left\{0, \pm g_{0}\right\}$ such that $g+h+k \in \Sigma$ for some $k \in\left\{0, g_{0},-g_{0}\right\}$ then $\mathcal{P}_{g} \mathcal{P}_{h} \neq 0$ if $k=0,\left\{\mathcal{P}_{g}, \mathcal{P}_{h}\right\} \neq 0$ if $k=g_{0}$ or $\left(\mathcal{P}_{g} \mathcal{P}_{h}\right) \mathcal{P}_{-g_{0}} \neq 0$ if $k=-g_{0}$. 
We recall that $\Sigma$ is called symmetric if $g \in \Sigma$ implies $-g \in \Sigma$. From now on we will suppose $\Sigma$ is symmetric.

We would like to note that the above concepts appear in a natural way in the study of any Poisson system. For instance, any graded Poisson structure associate to the Cartan grading of a semisimple finite dimensional Lie algebra gives rise to a $\Sigma$-multiplicative graded Poisson algebra with symmetric support and of maximal length. We also have, in the infinite-dimensional setting, that any graded Poisson structure $\mathcal{P}$ defined either on the split grading of a semisimple separable $L^{*}$-algebra, [24, 25], or on a semisimple locally finite split Lie algebra, [26], necessarily makes $\mathcal{P}$ a graded Poisson algebra with symmetric support, $\Sigma$-multiplicative and of maximal length. The Poisson algebras considered in [7, §3] are also examples of graded Poisson algebras with symmetric support of maximal length and $\Sigma$-multiplicative.

Lemma 7. Let $\mathcal{P}$ be centerless, $\Sigma$-multiplicative, of maximal length and with $\mathcal{P}_{\beta}$ tight for $\beta \in$ $\left\{0, \pm g_{0}, \pm 2 g_{0},-3 g_{0}\right\}$. If any couple of elements in $\Sigma$ are connected, then any nonzero ideal I of $\mathcal{P}$ satisfies $I=\mathcal{P}$.

Proof. Consider $I$ a nonzero ideal of $\mathcal{P}$ and write $I=\left(I \cap \mathcal{P}_{0}\right)+\left(I \cap \mathcal{P}_{g_{0}}\right)+\left(I \cap \mathcal{P}_{-g_{0}}\right) \oplus\left(\bigoplus_{g \in \Sigma_{I}}\left(I \cap \mathcal{P}_{g}\right)\right)$ where $\Sigma_{I}:=\left\{g \in \Sigma: I \cap \mathcal{P}_{g} \neq 0\right\}$. By the maximal length of $\mathcal{P}$ we can write

$$
I=\left(I \cap \mathcal{P}_{0}\right)+\left(I \cap \mathcal{P}_{g_{0}}\right)+\left(I \cap \mathcal{P}_{-g_{0}}\right) \oplus\left(\bigoplus_{g \in \Sigma_{I}} \mathcal{P}_{g}\right),
$$

being $\Sigma_{I} \neq \emptyset$ as consequence of Lemma 6 From here, we can take $g \in \Sigma_{I}$ being so

$$
0 \neq \mathcal{P}_{g} \subset I .
$$

For any $h \in \Sigma, h \neq \pm g$, the fact that $g$ and $h$ are connected allows us to fix a connection

$$
\left\{g_{1} \otimes 0, g_{2} \otimes k_{2}, \ldots, g_{n} \otimes k_{n}\right\}
$$

from $g$ to $h$. Consider $g_{1}=g, g_{2}$ and $g_{1}+g_{2}+k_{2}$. By $\Sigma$-multiplicativity and maximal length of $\mathcal{P}$ we obtain either $0 \neq \mathcal{P}_{g_{1}} \mathcal{P}_{g_{2}}=\mathcal{P}_{g_{1}+g_{2}}$ if $k_{2}=0$ or $0 \neq\left\{\mathcal{P}_{g_{1}}, \mathcal{P}_{g_{2}}\right\}=\mathcal{P}_{g_{1}+g_{2}+g_{0}}$ if $k_{2}=g_{0}$ or $0 \neq\left(\mathcal{P}_{g_{1}} \mathcal{P}_{g_{2}}\right) \mathcal{P}_{-g_{0}}=$ $\mathcal{P}_{g_{1}+g_{2}-g_{0}}$ if $k_{2}=-g_{0}$. From here, Equation (18) gives us that in any case

$$
0 \neq \mathcal{P}_{g_{1}+g_{2}+k_{2}} \subset I \text {. }
$$

We can argue in a similar way from $g_{1}+g_{2}+k_{2}, g_{3}$ and $g_{1}+g_{2}+k_{2}+g_{3}+k_{3}$ to get

$$
0 \neq \mathcal{P}_{g_{1}+g_{2}+k_{2}+g_{3}+k_{3}} \subset I \text {. }
$$

Following this process with the connection $\left\{g_{1} \otimes 0, g_{2} \otimes k_{2}, \ldots, g_{n} \otimes k_{n}\right\}$ we obtain that

$$
0 \neq \mathcal{P}_{g_{1}+g_{2}+k_{2}+g_{3}+k_{3}+\cdots+g_{n}+k_{n}} \subset I
$$

and so either $\mathcal{P}_{h} \subset I$ or $\mathcal{P}_{-h} \subset I$. That is, $0 \neq \mathcal{P}_{\epsilon_{h} h} \subset I$ for any $h \in \Sigma$ and some $\epsilon_{h} \in\{ \pm 1\}$.

Now, observe that we have showed that in case $h \notin \Sigma_{I}$ for some $h \in \Sigma$, then $-h \in \Sigma_{I}$. From here, if $-h+g_{0} \in \Sigma$, (resp. $-h-g_{0} \in \Sigma,-h-2 g_{0} \in \Sigma$ ), then by considering the set $-h, g_{0}, 0$, (resp. $-h,-g_{0}, 0$; $\left.-h,-g_{0},-g_{0}\right)$, the $\Sigma$-multiplicativity and maximal length of $\mathcal{P}$ give us now $\mathcal{P}_{-h+g_{0}} \subset I$, (resp. $\mathcal{P}_{-h-g_{0}} \subset I$, $\left.\mathcal{P}_{-h-2 g_{0}} \subset I\right)$. Hence, the fact $\mathcal{P}_{\alpha}$ is tight for any $\alpha \in\left\{0, g_{0},-g_{0}\right\}$ allows us to assert $\mathcal{P}_{0}+\mathcal{P}_{g_{0}}+\mathcal{P}_{-g_{0}} \subset I$.

Finally, the $\Sigma$-multiplicativity and maximal length of $\mathcal{P}$ together with the fact $\mathcal{P}_{0} \subset I$ allow us to assert that $\mathcal{P}_{h}=\mathcal{P}_{h} \mathcal{P}_{0} \subset I$ for any $h \in \Sigma$. Since

$$
\mathcal{P}=\mathcal{P}_{0}+\mathcal{P}_{g_{0}}+\mathcal{P}_{-g_{0}} \oplus\left(\bigoplus_{h \in \Sigma} \mathcal{P}_{h}\right) \subset I
$$

the proof is completed.

As consequence of Theorem 12 and Lemma 7 we can assert the next result.

Theorem 3. Let $\mathcal{P}$ be centerless, $\Sigma$-multiplicative, of maximal length and with $\mathcal{P}_{\beta}$ tight for $\beta \in$ $\left\{0, \pm g_{0}, \pm 2 g_{0},-3 g_{0}\right\}$. Then $\mathcal{P}$ is simple if and only if it has any couple of elements in $\Sigma$ connected.

Theorem 4. Let $\mathcal{P}$ be centerless, $\Sigma$-multiplicative, of maximal length and with $\mathcal{P}_{\beta}$ tight for $\beta \in$ $\left\{0, \pm g_{0}, \pm 2 g_{0},-3 g_{0}\right\}$. Then $\mathcal{P}$ is the direct sum of the family of its minimal ideals, each one being a simple Poisson color algebra of degree $g_{0}$ having all of the elements in its restricted support connected. 
Proof. By Corollary 1 we have that $\mathcal{P}=\bigoplus_{[g] \in \Sigma / \sim} \mathcal{I}_{[g]}$ is the direct sum of the ideals $\mathcal{I}_{[g]}$. We wish to apply Theorem 3 to any $\mathcal{I}_{[g]}$, so we have to verify that $\mathcal{I}_{[g]}$ is a centerless $\Sigma$-multiplicative Poisson color algebra of degree $g_{0}$ with maximal length, with $\left(\mathcal{I}_{[g]}\right)_{\beta}$ tight for $\beta \in\left\{0, \pm g_{0}, \pm 2 g_{0},-3 g_{0}\right\}$ and with all of the elements in its restricted support connected.

Since $\left(\mathcal{I}_{[g]}\right)_{\beta}=\mathcal{P}_{\beta,[g]}$ in case $\beta \in\left\{0, \pm g_{0}\right\}$ and $\mathcal{P}_{\beta}$ is tight, we clearly have $\left(\mathcal{I}_{[g]}\right)_{\beta}$ is tight for $\beta \in\left\{0, \pm g_{0}\right\}$. In case $\beta \in\left\{ \pm 2 g_{0},-3 g_{0}\right\} \backslash\left\{0, \pm g_{0}\right\}$ with $\beta \in \Sigma$, then $\beta \in[k]$ for a unique $[k] \in \Sigma / \sim$ and so $\left(\mathcal{I}_{[g]}\right)_{\beta}=0$ if $[g] \neq[k]$ and $\left(\mathcal{I}_{[k]}\right)_{\beta}=\sum_{h \in[k] \backslash\left\{ \pm n g_{0}: n \in 2,3\right\}}\left(\left\{\mathcal{P}_{h}, \mathcal{P}_{-h-g_{0}+\beta}\right\}+\mathcal{P}_{h} \mathcal{P}_{-h+\beta}\right)$. From here, taking into account $\operatorname{Remark} 1,\left(\mathcal{I}_{[g]}\right)_{\beta}$ is tight in any case.

We also have $\mathcal{I}_{[g]}$ is $\Sigma$-multiplicative as consequence of the $\Sigma$-multiplicativity of $\mathcal{P}$ and clearly $\mathcal{I}_{[g]}$ is of maximal length. Also observe that $\mathcal{I}_{[g]}$ is centerless as consequence of the fact $\left\{\mathcal{I}_{[g]}, \mathcal{I}_{[h]}\right\}+\mathcal{I}_{[g]} \mathcal{I}_{[h]}=0$ if $[g] \neq[h]$, (Theorem 2 ), and that $\mathcal{P}$ is centerless. Finally, since the restricted support of $\mathcal{I}_{[g]}$ is $[g]$, it is easy to verify that $[g]$ has all of its elements $[g]$-connected, (connected through elements contained in $[g] \cup\left\{0, \pm g_{0}\right\}$ ). From the above, we can apply Theorem 3 to any $\mathcal{I}_{[g]}$ so as to conclude $\mathcal{I}_{[g]}$ is simple. It is clear that the decomposition $\mathcal{P}=\underset{[g] \in \Sigma / \sim}{\bigoplus} \mathcal{I}_{[g]}$ satisfies the assertions of the theorem.

\section{REFERENCES}

[1] Akman, F.: A master identity for homotopy Gerstenhaber algebras. Comm. Math. Phys. 209, no. 1, (2000), 51-76.

[2] Avan, J. and Doikou, A.: Boundary Lax pairs from non-ultra-local Poisson algebras. J. Math. Phys. 50, no. 11, (2009), 113512,9 pp.

[3] Batalin, I.A. and Fradkin, E.S.: Quantization of gauge theories with linearly dependent generators. Phys. Rev. D (3), 28(10), (1983), $2567-2582$.

[4] Bautista, C. A Poincar-Birkhoff-Witt theorem for generalized Lie color algebras. J. Math. Phys. 39, no. 7, (1998), 38283843.

[5] Bruce, A.J.: From $L_{\infty}$-algebroids to higher Schouten/Poisson structures. Rep. Math. Phys. 67 , no. 2, (2011), 157-177.

[6] Calderón, A.J.: On the structure of graded Lie algebras. J. Math. Phys. 50, no. 10, (2009), 103513, 8 pp.

[7] Calderón, A.J.: On the structure of split non-commutative Poisson algebras. Linear and multilinear algebra. 60(7), (2012), 775-785.

[8] Calderón, A.J. and Sánchez, J.M.: On the structure of split Lie color algebras. Linear Algebra and its applications. 436(2), (2012), 307-315.

[9] Calderón, A.J. and Sánchez, J.M.: Weight modules over split Lie algebras. Modern Phys. Letters A. 28(5), (2013), 13500089 pp.

[10] Cattaneo, A.S., Fiorenza D. and Longoni R.: Graded Poisson algebras. In Francoise, J.P., Naber, G.L.; Tsun,T. S. Encyclopedia of Mathematical Physics. Amsterdam, 560-567.

[11] Draper, C., Martín, C. and Elduque. A.: Fine gradings on exceptional simple Lie superalgebras. Internat. J. Math. 22(12), (2011), $1823-1855$.

[12] Gibbons, J., Holm, D. and Tronci, D.: Cesare Geometry of Vlasov kinetic moments: a bosonic Fock space for the symmetric Schouten bracket. Phys. Lett. A 372, no. 23, (2008, 4184-4196.

[13] Henneaux M. and Teitelboim, C.: Quantization of gauge systems. Princenton University Press, Princenton, NJ, 1992.

[14] Hone, A.N. and Petrera, M.: Three-dimensional discrete systems of Hirota-Kimura type and deformed Lie-Poisson algebras. J. Geom. Mech. 1, no. 1, (2009), 55-85.

[15] Ikeda, N.: Deformation of graded Poisson (Batalin-Vilkovisky) structures. Poisson geometry in mathematics and physics, 147-161, Contemp. Math., 450, Amer. Math. Soc., Providence, RI, 2008.

[16] Khakimdjanov, Y. and Navarro, R.M.: Integrable deformations of nilpotent color Lie superalgebras. J. Geom. Phys. 61, no. 10, (2011), $1797-1808$.

[17] Kochetov, M.: Gradings on finite-dimensional simple Lie algebras. Acta Appl. Math. 108, no. 1, (2009), 101-127.

[18] Kosmann-Schwarzbach, Y.: Poisson and symplectic functions in Lie algebroid theory. Higher structures in geometry and physics, 243-268, Progr. Math., 287, Birkhuser/Springer, New York, 2011.

[19] Ngakeu, F.: Graded Poisson structures and Schouten-Nijenhuis bracket on almost commutative algebras. Int. J. Geom. Methods Mod. Phys. 9, no. 5, (2012), 1250042, 20 pp.

[20] Paufler, C.: A vertical exterior derivate in multisymplectic geometry and graded Poisson bracket for nontrivial geometries. Rep. Math. Phys.47(1), (2001), 101-119

[21] Piontkovski, D. and Silvestrov, S.D.: Cohomology of 3-dimensional color Lie algebras. J. Algebra 316, no. 2, (2007), $499-513$.

[22] Price, K.L.: A domain test for Lie color algebras. J. Algebra Appl. 7, no. 1, (2008), 81-90.

[23] Rottenberg, V.D.: Generalized superalgebras. Nucl. Phys. B. 139, (1978), 189-202.

[24] Schue J.R.: Hilbert Space methods in the theory of Lie algebras. Trans. Amer. Math. Soc. 95, (1960), 69-80.

[25] Schue J.R.: Cartan decompositions for $L^{*}$-algebras. Trans. Amer. Math. Soc. 98 (1961), 334-349.

[26] Stumme N.: The structure of Locally Finite split Lie Algebras. J. Algebra. 220 (1999), 664-693.

[27] Su, Y., Zhao, K. and Zhu, L.: Classification of derivation-simple color algebras related to locally finite derivations. J. Math. Phys. 45, no. 1, (2004), 525-536.

[28] Trostel, R.: Color analysis, variational selfadjointness, and color Poisson (super)algebras. J. Math. Phys. 25, no. 11, (1984), 31833189.

[29] Vankerschaver, J., Yoshimura, H. and Leok, M.: On the geometry of multi-Dirac structures and Gerstenhaber algebras. J. Geom. Phys. 61 , no. 8, (2011), 1415-1425. 
[30] Wei, Y. and Wettig, T.: Bosonic color-flavor transformation for the special unitary group. J. Math. Phys. 46, no. 7, (2005), 072306, 25 pp.

[31] Xu, P. Gerstenhaber algebras and $B V$-algebras in Poisson geometry. Comm. Math. Phys. 200, no. 3, (1999), 545-560.

[32] Zhang, Q. and Zhang, Y.: Derivations and extensions of Lie color algebra. Acta Math. Sci. Ser. B Engl. Ed. 28, no. 4, (2008), $933-948$.

[33] Zhang, X. and Zhou, J.: Derivation simple color algebras and semisimple Lie color algebras. Comm. Algebra 37, no. 1, (2009), $242-257$.

[34] Zhao, K.: Simple Lie color algebras from graded associative algebras. J. Algebra 269, no. 2, (2003), 439-455

Antonio J. Calderón Martin. Department of Mathematics. Univesity of Cádiz. 11510 Puerto Real, Cádiz. (Spain), e-mail: ajesus.calderon@uca.es

D. Mame Cheikh. Department of Mathematics. University of Dakar. e-mail: dioufmamecheikh@gmail.com 